\title{
Erratum to: Non-equilibrium thermodynamics and maximum entropy production in the earth system: Applications and implications
}

\author{
Axel Kleidon
}

Published online: 9 November 2010

(C) Springer-Verlag 2010

\section{Erratum to: Naturwissenschaften}

\section{DOI 10.1007/s00114-009-0509-x}

Figure 2 contains two aspects that are incorrect: First, the figure does not show the entropy production by mixing $\sigma_{a}$, but rather the increase of entropy production by mixing due to the presence of heat transport, that is, $\Delta \sigma_{a}=\sigma_{a}\left(F_{a b}\right)-\sigma_{a}$ $\left(\mathrm{F}_{\mathrm{ab}}=0\right)$ where $\mathrm{F}_{\mathrm{ab}}$ is the heat transport from box A to $\mathrm{B}$. Second, the entropy of the whole system was computed in an incorrect way. The corrected plot is shown below.

The errors do not affect the main messages and conclusions of the paper. It does, however, affect the interpretation of the relationship of a state of Maximum Entropy Production (MEP) and a state of low entropy or the extent of disequilibrium. A corrected explanation of this relationship is given by Kleidon ("Life, hierarchy, and the thermodynamic machinery of planet Earth", published online in Physics of Life Reviews, doi: 10.1016/j.plrev.2010.10.002.)

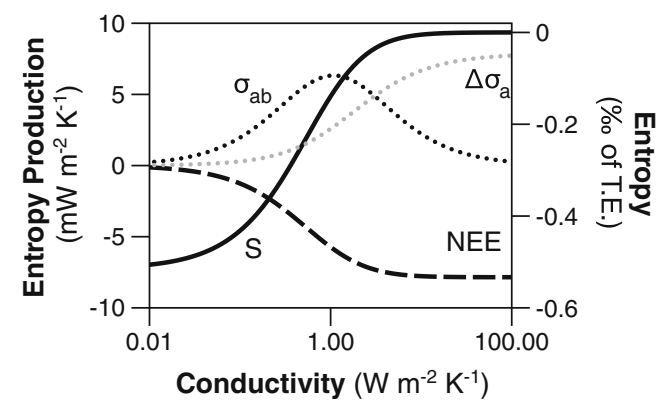

Figure 2 Sensitivity of the model to the flux $F_{a b}$ by varying its conductivity $\mathrm{k}$ in terms of entropy $\mathrm{S}$ (expressed in relation to a state of thermodynamic equilibrium), net entropy exchange NEE, and the increase in entropy production by mixing $\Delta \sigma_{\mathrm{a}}$ within box A and by heat transport $\Delta \sigma_{\mathrm{ab}}$ org/10.1007/s00114-009-0509-x.

\section{A. Kleidon $(\square)$}

Max-Planck-Institut für Biogeochemie,

Postfach 1001 64,

07701 Jena, Germany

e-mail: akleidon@bgc-jena.mpg.de 